\title{
A multi-objective programming approach to Weibull parameter estimation
}

\author{
Emre Koçak ${ }^{* 1}$ (D), Ecem Demir Yurtseven ${ }^{2,3}$ (D), H. Hasan Örkc ̈̈ ${ }^{1}$ (D) \\ ${ }^{1}$ Department of Statistics, Faculty of Science, Gazi University, Ankara, Turkey \\ ${ }^{2}$ Rector's Office, Sivas Cumhuriyet University, Sivas, Turkey \\ ${ }^{3}$ Graduate School of Natural and Applied Sciences, Gazi University, Ankara, Turkey
}

\begin{abstract}
Weibull distribution is widely used in various areas such as life tables, failure rates, and definition of wind speed distribution. Therefore, parameter estimation for the Weibull distribution is an important problem in many real data applications. The least square (LS), the weighted least square (WLS) and the maximum likelihood (ML) are the most popular methods for the parameter estimation in the Weibull distribution. In this study, based on the LS, WLS and ML estimation methods, a multi-objective programming approach is proposed for the parameter estimation of two-parameter Weibull distribution. This new approach evaluates together LS, WLS and ML methods in the estimation process. NSGAII method, which is a multi-objective heuristic optimization method, is used to solve the proposed multi-objective estimation model. To evaluate the applicability and performance of the proposed approach, a detailed Monte Carlo simulation study based on deficiency criteria and a real data application are designed. The results illustrated that the proposed multi-objective programming approach provides quite accurate parameter estimates for the two parameter Weibull distribution with respect to deficiency criteria.
\end{abstract}

Mathematics Subject Classification (2020). 58E17, 62F07, 62F10

Keywords. Weibull parameter estimation, multi-objective programming, NSGA-II

\section{Introduction}

It is a well-known fact that the Weibull distribution family, besides being flexible due to imitating various distributions like the exponential or normal, can very well fit a wide field of experimental observations. In addition to demonstrating a wide range of shapes for density and hazard functions, it is also an important distribution used to analyze the reliability of different types of systems. This distribution can be applied to two or three parameters depending on the field of use and it is used in quality control, analysis of life tables, failure rates, definition of wind speed distribution, and financial applications.

\footnotetext{
*Corresponding Author.

Email addresses: emrekocak@gazi.edu.tr (E. Koçak), ecemdemir@cumhuriyet.edu.tr (E. Demir Yurtseven), hhorkcu@gazi.edu.tr (H.H. Örkcü)

Received: 09.04.2021; Accepted: 14.12.2021
} 
The cumulative distribution function and the corresponding probability density function of the two-parameter Weibull distribution is given by

$$
F_{x}(x)=1-e^{-\left(\frac{x}{\eta}\right)^{\theta}}, \quad x>0, \eta>0, \theta>0
$$

and

$$
f_{x}(x)=\frac{\theta}{\eta}\left(\frac{x}{\eta}\right)^{\theta-1} e^{-\left(\frac{x}{\eta}\right)^{\theta}}, \quad x>0, \eta>0, \theta>0,
$$

respectively. In Equations (1.1) and (1.2), $\eta$ is scale parameter and $\theta$ is shape parameter.

Because of the wide applications area, it is very important to determine the best parameter estimation method for this distribution. Successful applications of the Weibull distribution depend on having acceptable statistical estimates of its hardly predictable parameters. Several methods have been used for estimating the parameters of the Weibull distribution. Various estimation methods for the Weibull parameters have been proposed by many authors until today. The least squares (LS) method, weighted least squares (WLS) method, maximum likelihood (ML) method, moments method and Bayesian methods are used to estimate the parameters of the Weibull distribution. The ML method is the most popular way of estimating the parameters of the density function from observed data for distributions. In addition to providing simple closed form solutions for estimates, the LS method is computationally easier. Abbasi et al. [1] used the simulated annealing (SA) method to maximize the likelihood function value of the Weibull distribution and estimate the parameters. Moreover, Abbasi et al. [2] proposed a hybrid variable neighbourhood search and SA method to get better the performance of the SA method. Örkcü et al. [15] presented a comprehensive study of different particle swarm optimization (PSO) variants in the parameter estimation problem of the three-parameter Weibull distribution. The ML of Weibull distribution parameters were taken into account using PSO by [3]. Markovic et al. [13] studied the nonlinear WLS estimation for the three-parameter Weibull distribution. Hossain and Howlader [10] made comparisons between various LS and ML estimators for the shape parameter and complete samples. Luus and Jammer [12] showed that ML gave the most reliable parameter estimation compared to the LS method. Davies [7] investigated the unbiased estimation of the Weibull scale parameter with a function of $n$ samples using linear LS. While Lei [11] and Chu and Ke [5] dealt with LS and ML methods in their studies, Pobacikova and Sedliackova [16], Datsiou and Overend [6] and, Nassar et al. [14] discussed the WLS method in addition to these methods.

LS, WLS and ML methods are based on different theoretical bases and have different properties. While the likelihood function is maximized in ML based methods, the error function is tried to be minimized in LS and WLS based methods. In either case, there is only one objective function that is maximized or minimized. As an optimization tool, classical approaches based on derivative are used as well as heuristic approaches such as genetic algorithm, simulated annealing, and particle swarm optimization. LS, WLS and ML methods can give different estimation results within the framework of their theoretical basis. This study, based on the LS, WLS and ML estimation methods, proposes using the multi-objective programming approach for the estimation of parameter of Weibull distribution. In this way, the parameter estimation process in LS, WLS and ML methods will be evaluated together, and it is aimed to obtain better estimation results. In this study, Genetic Algorithm based Non-dominated Sorting Genetic Algorithm II (NSGAII) method, which is a multi-objective heuristic approach, is used to solve the formed multi-objective programming estimation model. LS-WLS, LS-ML, WLS-ML and LS-WLSML cases have been taken into consideration as the multi-objective estimation model and the formed multi-objective programming estimation approaches have been compared with the classical LS, WLS and ML methods. The results show that the multi-objective optimization model, which takes LS, WLS and ML estimation procedures into account together, gives more successful results than classical approaches in parameter estimation. 
To the best of our knowledge, this is the first study to use the NSGA-II method for estimating the parameters of the two-parameter Weibull distribution.

The remainder of the paper is organized as follows. In Section 2, the LS, the WLS and the ML estimations for two-parameter Weibull distribution presented, and the NSGA-II method is introduced. A comprehensive Monte Carlo (MC) simulation study is carried out and its results are given in Section 3. In Section 4, the application of the proposed approach is examined on real life data. Section 5 summarizes the results of this work and draws conclusions.

\section{Parameter estimation for two-parameter Weibull distribution by NSGA- II method}

In this section, classical LS, WLS and ML estimation methods are briefly introduced and the proposed multi-objective approach that takes these methods into account is presented. In addition, the NSGA-II algorithm used in the solution of the proposed multi-objective programming model is introduced.

\subsection{Estimation methods}

The notions of LS, WLS and ML estimators of parameters of the two-parameter Weibull distribution are discussed in this section.

Let $x_{1}, x_{2}, \ldots, x_{n}$ be a random sample of size $n$ drawn from a probability density function in Equation (1.2).

2.1.1. Least square method. To convert the cumulative distribution function to a linear function, Equation (2.1) is obtained by taking twice the logarithm of Equation (1.1).

$$
\ln [-\ln (1-F(x))]=\theta \ln x-\theta \ln \eta
$$

If $Y=\ln [-\ln (1-F(x))], \beta_{0}=-\theta \ln \eta, \beta_{1}=\theta$ and $X=\ln x$ transformations are made, Equation (2.2) can be written as follows:

$$
Y=\beta_{0}+\beta_{1} X
$$

Considering $X_{(1)}, X_{(2)}, \ldots, X_{(n)}$ as the order statistics of $X_{1}, X_{2}, \ldots, X_{n}$ and $x_{(1)}, x_{(2)}, \ldots, x_{(n)}$ as observed ordered observations, the mean rank given in Equation (2.3) is used to estimate the values of the cumulative distribution function in Equation (1.1).

$$
\hat{F}\left(x_{(i)}\right)=\frac{i}{n+1}, \quad i=1,2, \ldots, n
$$

Here, $i$ denotes $i^{\text {th }}$ smallest value of $x_{(1)}, x_{(2)}, \ldots, x_{(n)}$. By minimizing the function in Equation (2.4), the estimates $\hat{\beta}_{0}$ and $\hat{\beta}_{1}$ of the regression parameters $\beta_{0}$ and $\beta_{1}$ are obtained as

$$
\Psi\left(\beta_{0}, \beta_{1}\right)=\sum_{i=1}^{n}\left(Y_{i}-\beta_{0}-\beta_{1} \ln x_{(i)}\right)^{2} .
$$

2.1.2. Weighted least square method. By minimizing the function in Equation (2.5), the estimates $\hat{\beta}_{0}$ and $\hat{\beta}_{1}$ of the regression parameters $\beta_{0}$ and $\beta_{1}$ are obtained as

$$
\Psi\left(\beta_{0}, \beta_{1}\right)=\sum_{i=1}^{n} w_{i}\left(Y_{i}-\beta_{0}-\beta_{1} \ln x_{(i)}\right)^{2} .
$$

The weight factor $w_{i}$ in Equation (2.6) suggested by [4] is formulated as follows:

$$
w_{i}=\left[\left(1-\hat{F}\left(x_{(i)}\right)\right) \ln \left(1-\hat{F}\left(x_{(i)}\right)\right)\right]^{2}, \quad i=1,2, \ldots, n .
$$


2.1.3. Maximum likelihood method. Maximum likelihood estimators of the twoparameter Weibull distribution in Equation (1.2) are found by maximizing the likelihood or log-likelihood function in Equations (2.7) and (2.8), respectively.

$$
L=\prod_{i=1}^{n} f_{x}\left(x_{i} ; \eta, \theta\right)=\prod_{i=1}^{n} \frac{\theta}{\eta}\left(\frac{x}{\eta}\right)^{\theta-1} e^{-\left(\frac{x_{i}}{\eta}\right)^{\theta}}
$$

Its logarithm is as follows:

$$
\ln L=n \ln \left(\frac{\theta}{\eta}\right)+\sum_{i=1}^{n}\left[-\left(\frac{x_{i}}{\eta}\right)^{\theta}+(\theta-1) \ln \left(\frac{x_{i}}{\eta}\right)\right] .
$$

\subsection{Proposed multi-objective optimization approach}

Proposed multi-objective optimization approach was created using the functions of LS, WLS and ML methods. While it is desired to make the function minimum in LS and WLS methods, it is desired to make maximum in ML method. Therefore, the additive inverse of the ML function is taken.

The models created for LS-WLS, LS-ML, WLS-ML and LS-WLS-ML are specified in Equations (2.9) - (2.12), respectively.

$$
\begin{aligned}
& \text { Minimize }\left\{\begin{array}{l}
\sum_{i=1}^{n}\left(\ln [-\ln (1-F(x))]+\theta \ln \eta-\theta \ln x_{(i)}\right)^{2} \\
\sum_{i=1}^{n} w_{i}\left(\ln [-\ln (1-F(x))]+\theta \ln \eta-\theta \ln x_{(i)}\right)^{2}
\end{array}\right. \\
& \text { Minimize }\left\{\begin{array}{l}
\sum_{i=1}^{n}\left(\ln [-\ln (1-F(x))]+\theta \ln \eta-\theta \ln x_{(i)}\right)^{2} \\
-n \ln \left(\frac{\theta}{\eta}\right)-\sum_{i=1}^{n}\left[-\left(\frac{x_{i}}{\eta}\right)^{\theta}+(\theta-1) \ln \left(\frac{x_{i}}{\eta}\right)\right]
\end{array}\right. \\
& \text { Minimize }\left\{\begin{array}{l}
\sum_{i=1}^{n} w_{i}\left(\ln [-\ln (1-F(x))]+\theta \ln \eta-\theta \ln x_{(i)}\right)^{2} \\
-n \ln \left(\frac{\theta}{\eta}\right)-\sum_{i=1}^{n}\left[-\left(\frac{x_{i}}{\eta}\right)^{\theta}+(\theta-1) \ln \left(\frac{x_{i}}{\eta}\right)\right]
\end{array}\right. \\
& \text { Minimize }\left\{\begin{array}{l}
\sum_{i=1}^{n}\left(\ln [-\ln (1-F(x))]+\theta \ln \eta-\theta \ln x_{(i)}\right)^{2} \\
\sum_{i=1}^{n} w_{i}\left(\ln [-\ln (1-F(x))]+\theta \ln \eta-\theta \ln x_{(i)}\right)^{2} \\
-n \ln \left(\frac{\theta}{\eta}\right)-\sum_{i=1}^{n}\left[-\left(\frac{x_{i}}{\eta}\right)^{\theta}+(\theta-1) \ln \left(\frac{x_{i}}{\eta}\right)\right]
\end{array}\right.
\end{aligned}
$$

In general, the main purpose of multi-objective optimization problems is to determine the variable values that will give the best value to the objective functions, so there are various approaches developed for the solution of the problem. In approaches such as dimension reduction, a solution is tried to be reached by transforming the addressed objective functions into a single objective function. However, the result obtained is optimum for one objective function but not for other functions. Therefore, there are alternative solution sets, and these solutions are called Pareto optimal solutions instead of a single optimal solution, which is called the ideal solution in the solution of the multi-objective optimization problem.

In addition to offering a wide set of solutions, there are various approaches to obtain Pareto solutions in which there is a compromised solution for all objective functions discussed. Multi-objective metaheuristic methods are important methods in obtaining Pareto 
solutions because they produce many solutions, and they do not use derivative calculations but provide a good approach to Pareto optimal solutions and can be easily applied to optimization problems.

\subsection{Non-dominated sorting genetic algorithm II (NSGA-II)}

Genetic Algorithm based NSGA-II, besides being a multi-objective metaheuristic method, is one of the most effective methods used in obtaining the Pareto solution set. The superiority of the NSGA-II method over other multi-objective genetic algorithms is its fast non-dominated sorting and crowding distance. For these reasons, by obtaining different solutions in the Pareto solution set, it reaches real Pareto values in a faster time $[8,9]$. Algorithm steps for fast non-dominant sorting and crowding distance are given in Tables 1 and $2[9]$.

Table 1. Fast non-dominated sort algorithm.

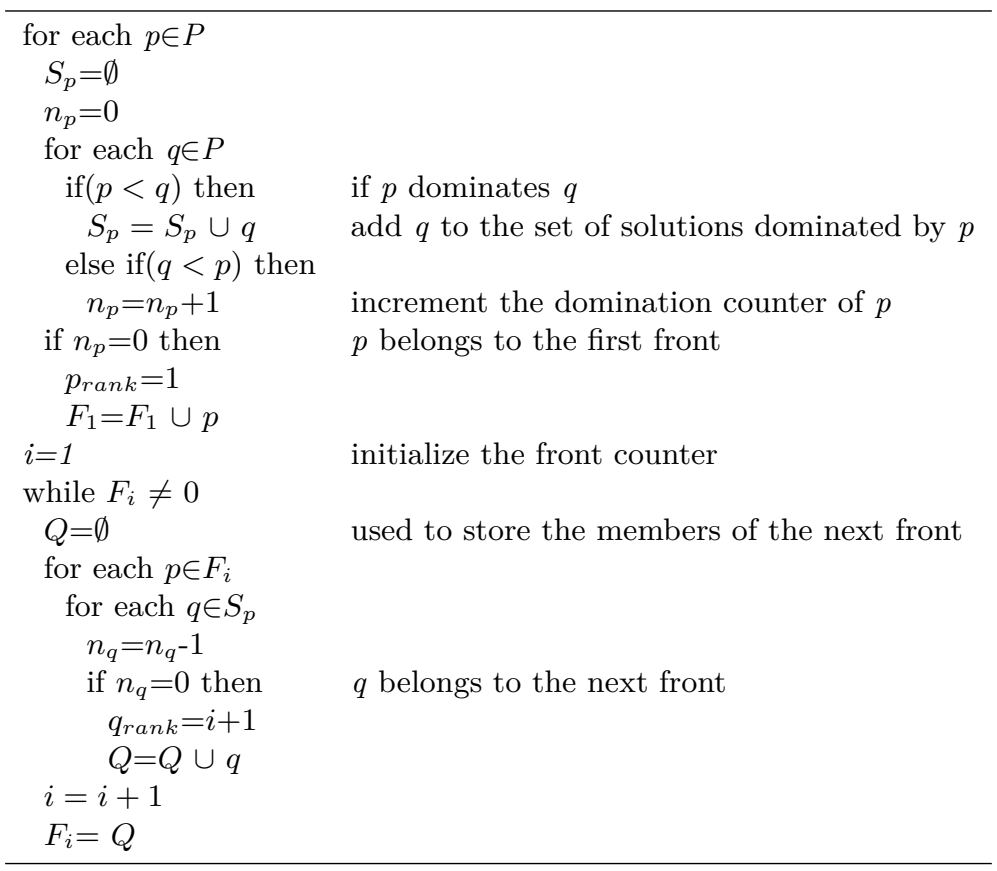

Table 2. Crowding distance assignment algorithm.

\begin{tabular}{ll}
\hline$l=|I|$ & number of solution in $I$ \\
for each $i$, set $I[i]_{\text {distance }}=0$ & initalize distance \\
for each objective $m$ & \\
$I=\operatorname{sort}(I, m)$ & sort using each objective value \\
$I[1]_{\text {distance }}=I[l]_{\text {distance }}=\infty$ & so that boundary pointsare always selected \\
$\begin{array}{c}\text { for } i=2 \text { to }(l-1) \\
(I[i+1] . m-I[i-1] . m)\end{array}$ & for all other points \\
$\quad\left(f_{m}^{\text {max }}-f_{m}^{\text {min }}\right)$ & \\
\hline
\end{tabular}

In the NSGA-II method based on population-based searches, the search is started with a set of solutions, each representing a possible solution to the problem, and better solutions are tried to be obtained from the existing solution set. The best individuals of the current population are selected, and a new population is created by crossing and mutation operators. It continues to create a population for the predetermined number of iterations. Algorithm steps and procedure for NSGA-II are given in Table 3 and in Figure $1[8,9]$. 
For more details of this procedure, the readers are encouraged to refer to the original papers $[8,9]$.

Table 3. NSGA-II algorithm.

\begin{tabular}{ll}
\hline$R_{t}=P_{t} \cup Q_{t}$ & combine parent and offspring population \\
$F=$ fast non-dominated sort $\left(R_{t}\right)$ & $F=\left(F_{1}, F_{2}, \ldots\right)$ all nondominated fronts of $R_{t}$ \\
$P_{t+1}=\emptyset$ and $i=1$ & \\
until $P_{t+1}=F_{i} \leq N$ & until the parent population is filled \\
$\quad$ crowding distance assignment $\left(F_{i}\right)$ & calculate crowding distance in $F_{i}$ \\
$P_{t+1}=P_{t+1} \cup F_{i}$ & include $i$ th nondominated front in the parent pop \\
$i=i+1$ & check the next front for inclusion \\
Sort $\left(F_{i}, \prec_{n}\right)$ & sort in descending order using $\prec_{n}$ \\
$P_{t+1}=P_{t+1} \cup F_{i}\left[1:\left(N-\left|P_{t+1}\right|\right)\right]$ & choose the first $\left[1:\left(N-\left|P_{t+1}\right|\right)\right]$ elements of $F_{i}$ \\
$Q_{t+1}=$ make new pop $\left(P_{t+1}\right)$ & use selection, crossover and mutation \\
& to create a new population $Q_{t+1}$ \\
$t=t+1$ & increment the generation counter
\end{tabular}

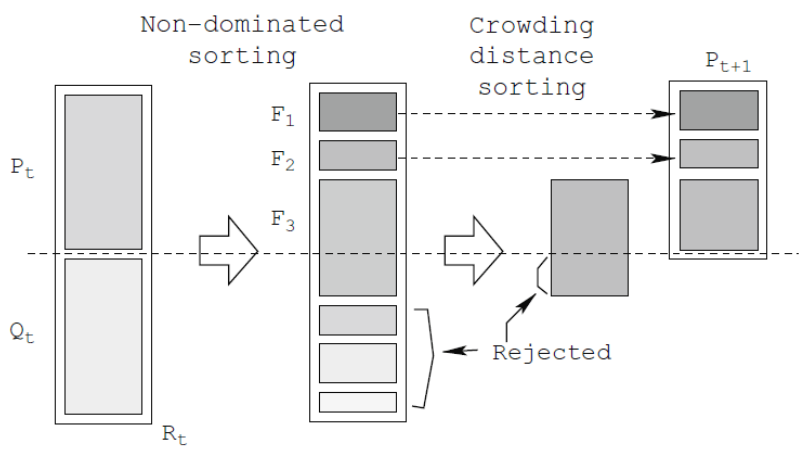

Figure 1. NSGA-II procedure.

\section{Simulation study}

In this section, a comprehensive MC simulation study for examining the performance of the proposed LS, WLS, ML, LS-WLS, LS-ML, WLS-ML and LS-WLS-ML multi-objective programming estimation methods was performed. Performances of the different case are compared with respect to their Deficiency Criterion (Def), see [17]. It is an important scale used to test the efficiency of methods used for parameter estimation [18]. It is defined as given in Equation (3.1). In addition, the mean squared error (MSE) values of the parameters used in the calculation of Def criteria are as given in Equations (3.2) and (3.3).

$$
\operatorname{Def}(\hat{\eta}, \hat{\theta})=\operatorname{MSE}(\hat{\eta})+\operatorname{MSE}(\hat{\theta}),
$$

where

$$
\operatorname{MSE}(\hat{\eta})=\operatorname{Var}(\hat{\eta})+\operatorname{Bias}^{2}(\hat{\eta})
$$

and

$$
\operatorname{MSE}(\hat{\theta})=\operatorname{Var}(\hat{\theta})+\operatorname{Bias}^{2}(\hat{\theta}) .
$$

Furthermore, $E^{2}$ value is used in comparison of parameter estimation results [2]. The best solution among Pareto points in the parameter space for LS-WLS, LS-ML, WLS-ML and LS-WLS-ML is obtained by choosing the point corresponding to the best $E^{2}$ value of 
the prediction points. The $E^{2}$ value given in Equation (3.4) is calculated as the sum of the differences between the real parameter values and the estimated parameter values.

$$
E^{2}=\sum_{i=1}^{n}[\gamma-\hat{\gamma}]^{2}
$$

Real parameter values for the two-parameter Weibull distribution are to be $(\eta, \theta)=$ $(2,2),(\eta, \theta)=(2,3)$ and $(\eta, \theta)=(3,2)$ respectively. The sample size is taken $\mathrm{n}=100,500$, 1000 and 2500. LS, WLS, ML, LS-WLS, LS-ML, WLS-ML and LS-WLS-ML estimates of the parameters for the two-parameter Weibull distribution are calculated using the NSGA-II algorithm.

Parameter values for the NSGA-II algorithm are considered as Crossover Fraction $=$ 0.8 and Pareto Fraction $=0.35$. Population Size (Pop) is also considered to be 50, 100 and 250 respectively while the Search Space for $\eta$ and $\theta$ is chosen as $(0$, inf).

The simulated mean and Def values for $(\eta, \theta)=(2,2),(\eta, \theta)=(2,3),(\eta, \theta)=(3,2)$ are given respectively in Tables $4-6$. The simulation results show that the case formed by LSWLS-ML estimators has the best Def value for all cases. However, this case does not give the best results in any case when compared to its parameter estimation. The reasons for this situation are that achieving objectives in cases with more than one objective function is difficult to compare to cases with an objective function, and the spread of predicted values is less in multi-objective function cases when estimating parameters. However, parameter estimation values in different situations are very close to each other. Regarding this situation, the distributions of the parameter estimates obtained by MC are shown in Figures $2-8$ for the parameter $(\eta, \theta)=(2,2)$.

When the simulation results are examined in terms of parameter estimates, it has been observed that the WLS-ML case makes the best parameter estimation for most cases. While $\mathrm{n}=100$ and $\mathrm{n}=500$, the best parameter estimation in all cases except Pop $=50$ has been obtained with the WLS-ML case. In addition, the best estimation was again obtained with WLS-ML when Pop $=250$ for all parameter values, excluding $(\eta, \theta)=(2$, 2 ) and $n=2500$. Apart from these cases, the best parameter estimates were obtained with WLS and ML. Since it does not have the best predictor case for any situation, a case including LS method is weak when compared to others in parameter estimation.
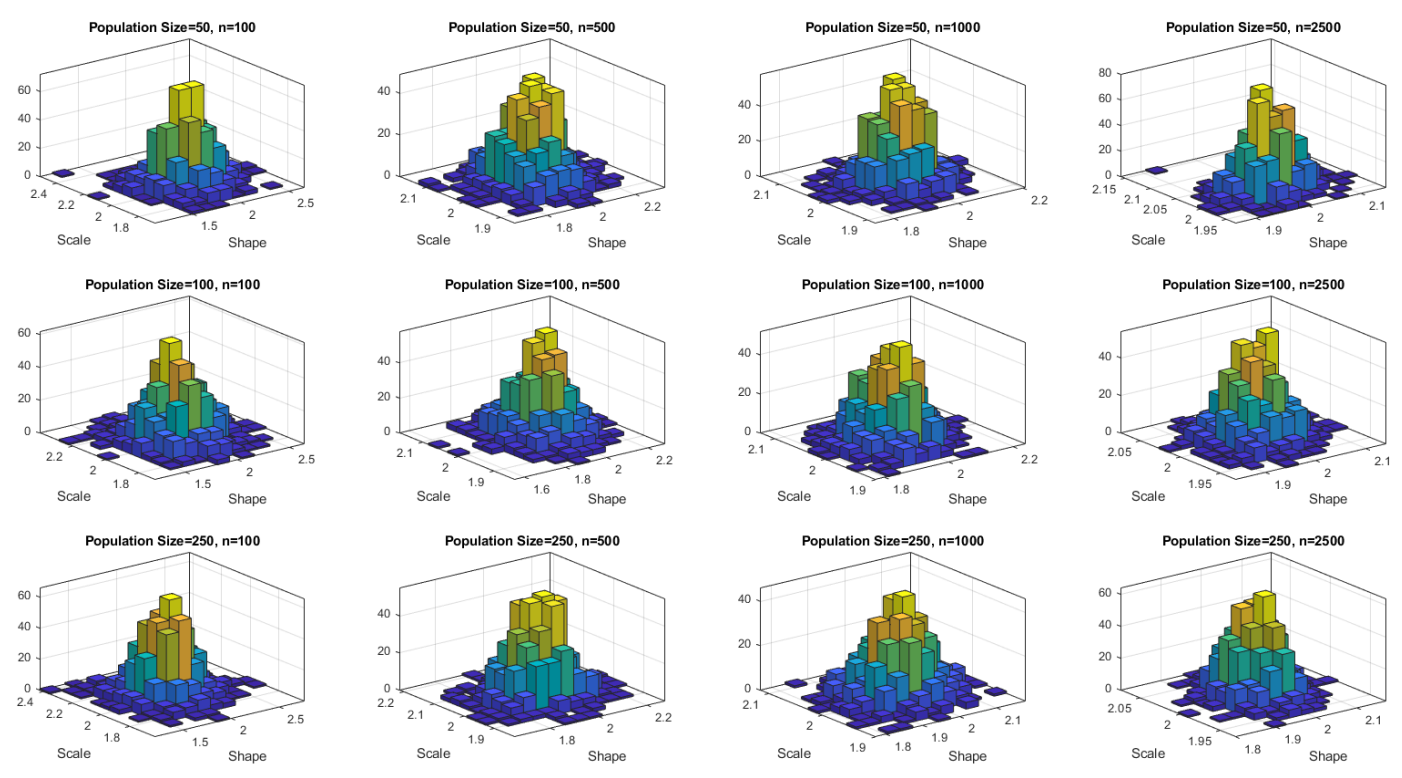

Figure 2. Distribution of parameter estimations obtained by MC simulation for $(\eta, \theta)=(2,2) /$ LS. 

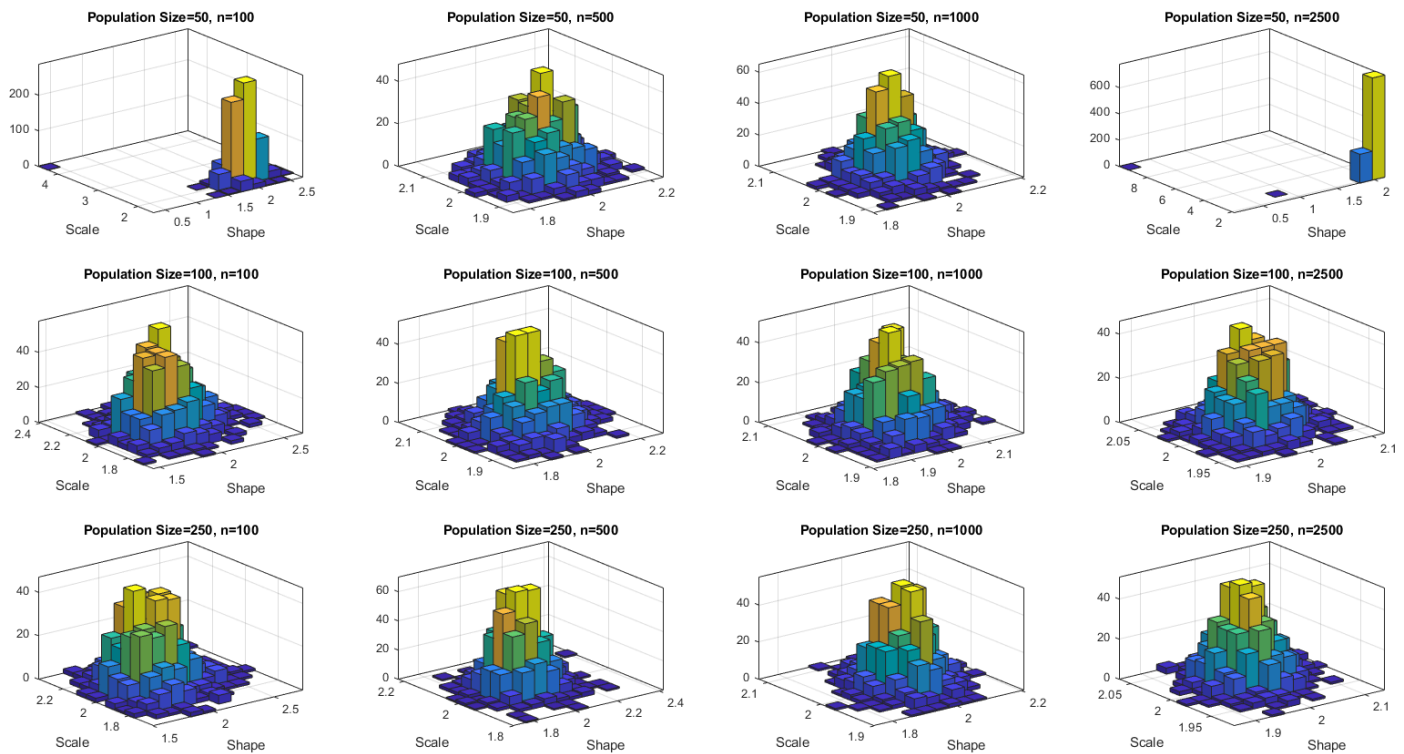

Figure 3. Distribution of parameter estimations obtained by MC simulation for $(\eta, \theta)=(2,2) /$ WLS.
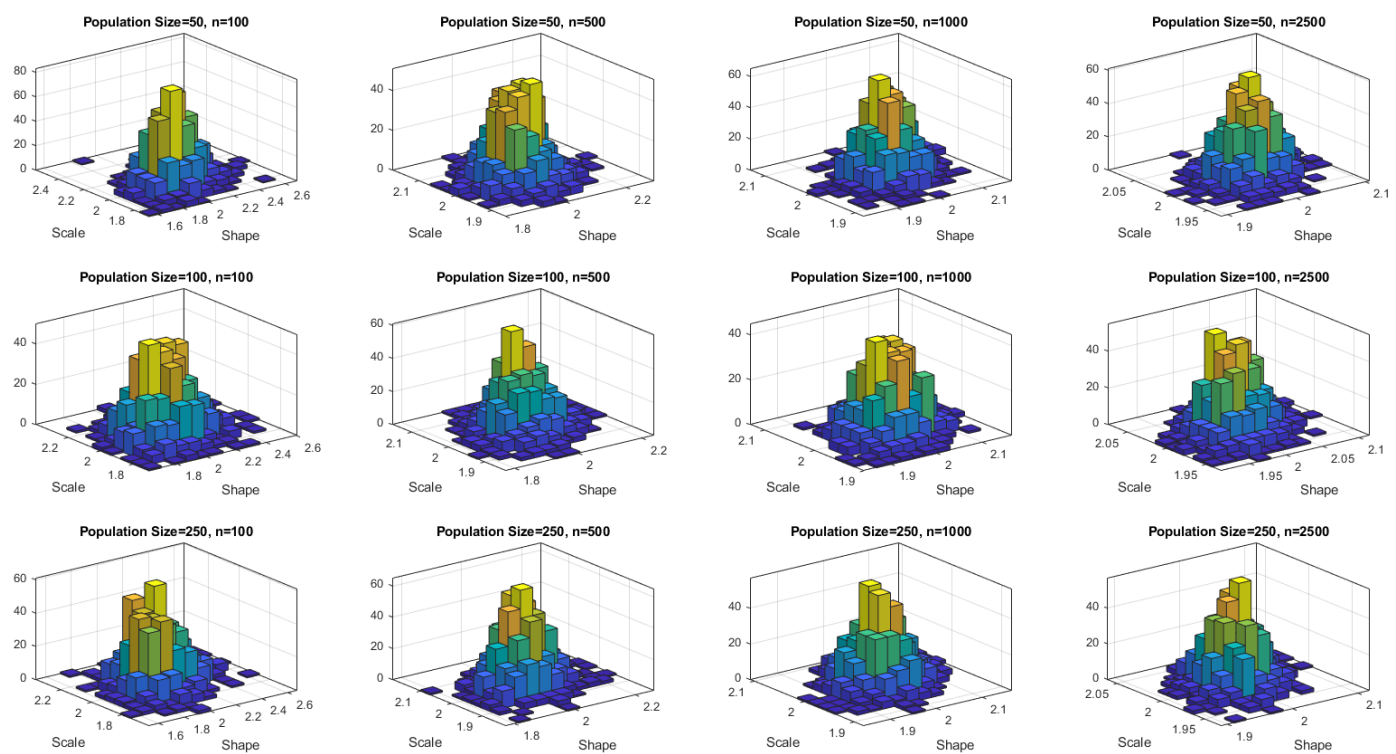

Figure 4. Distribution of parameter estimations obtained by $\mathrm{MC}$ simulation for $(\eta, \theta)=(2,2) /$ ML. 

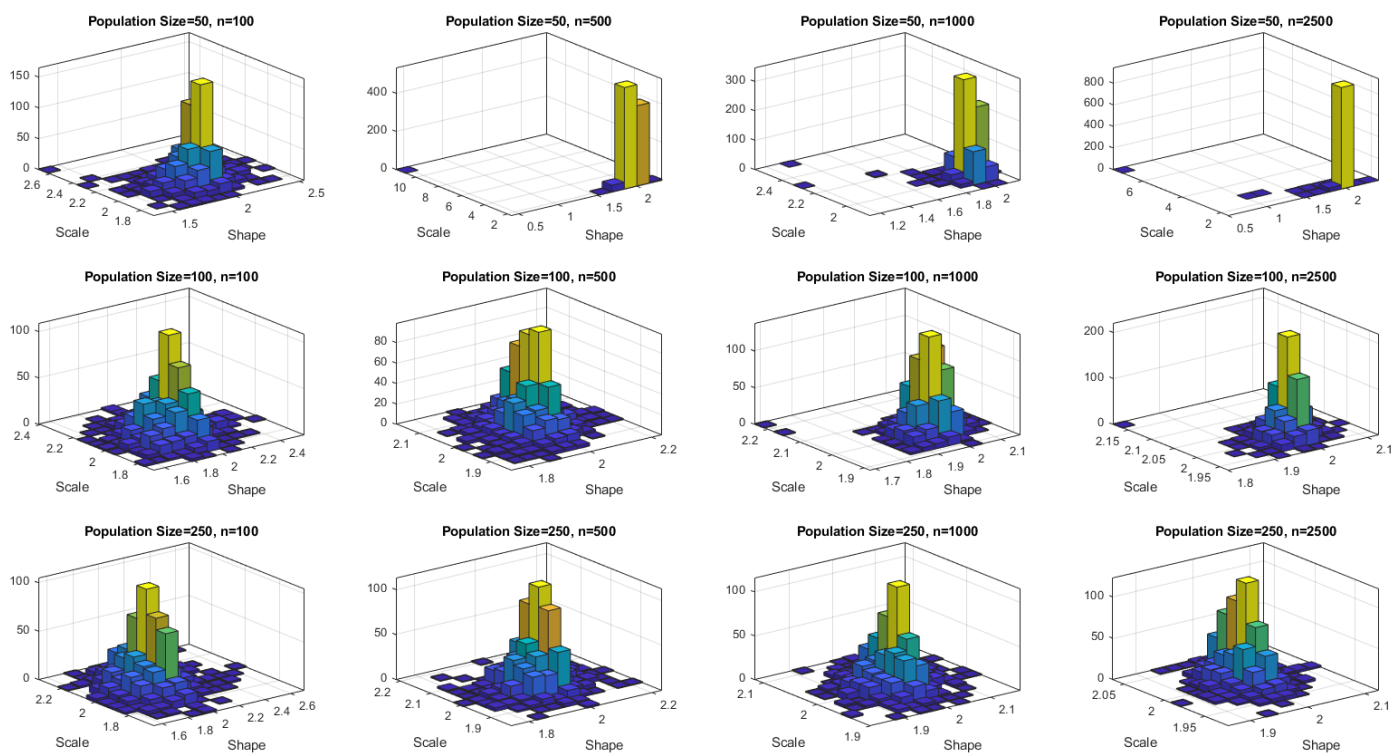

Figure 5. Distribution of parameter estimations obtained by MC simulation for $(\eta, \theta)=(2,2) /$ LS-WLS.
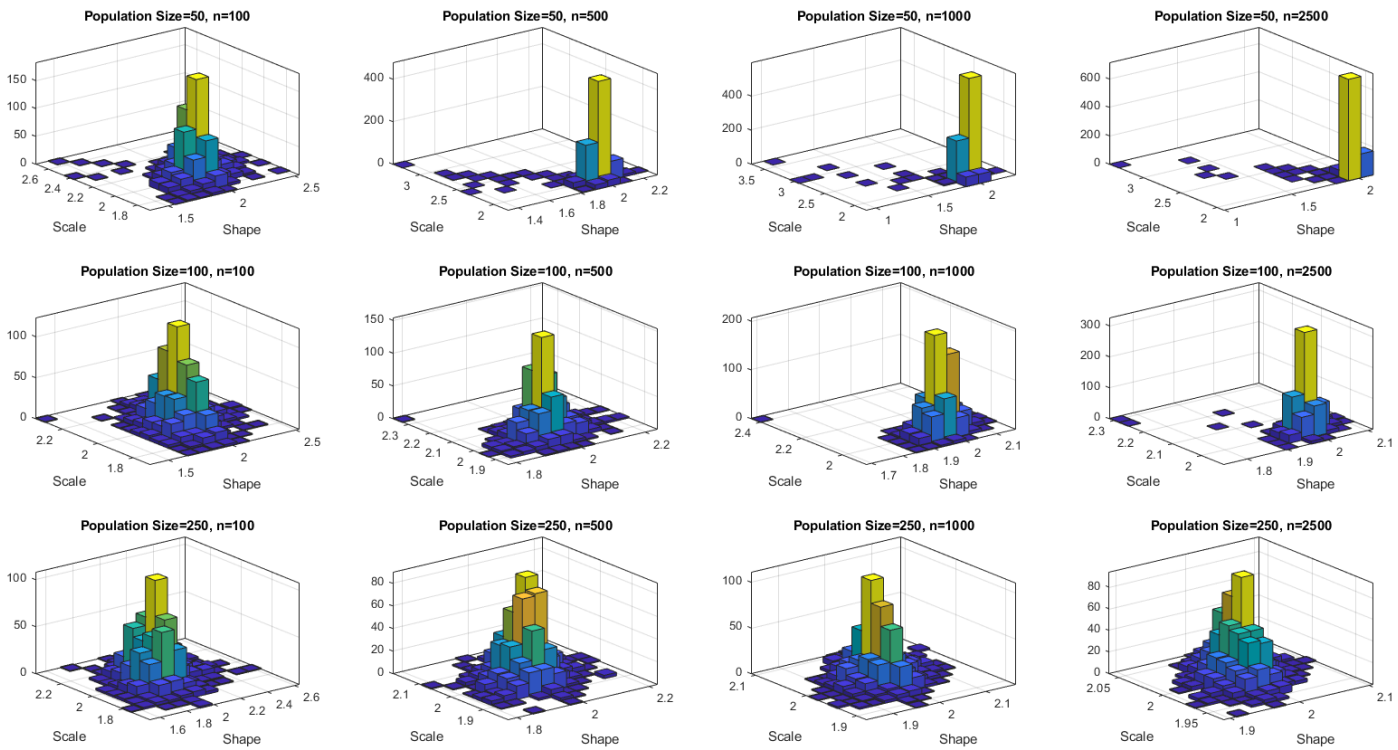

Figure 6. Distribution of parameter estimations obtained by MC simulation for $(\eta, \theta)=(2,2) /$ LS-ML 

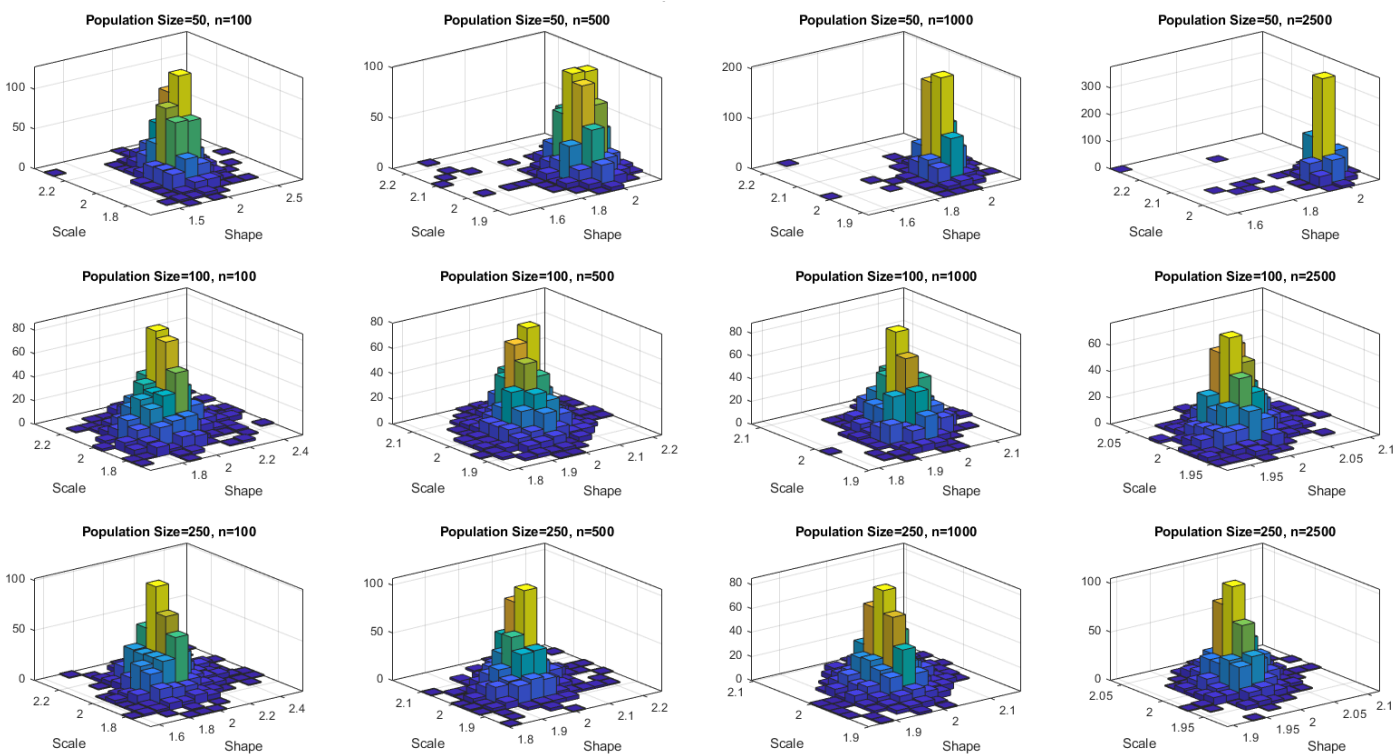

Figure 7. Distribution of parameter estimations obtained by $\mathrm{MC}$ simulation for $(\eta, \theta)=(2,2) /$ WLS-ML.
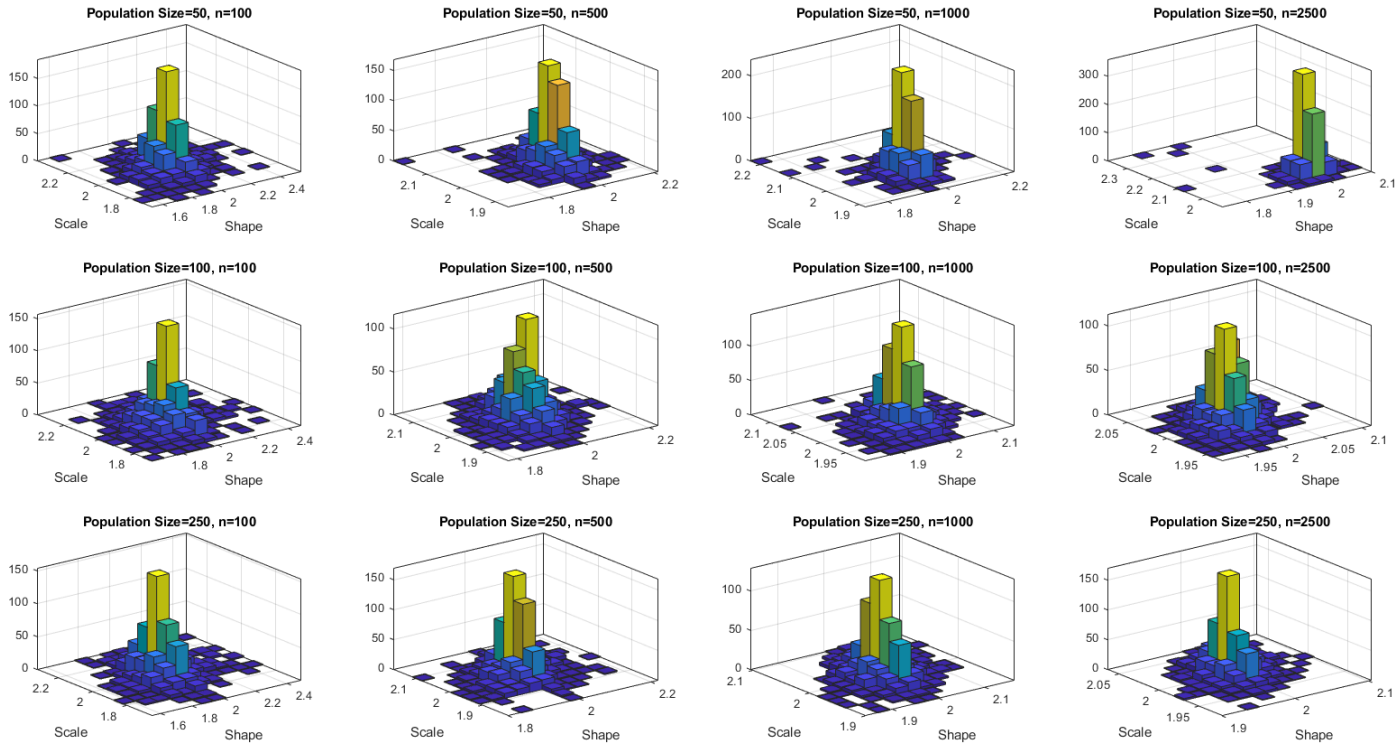

Figure 8. Distribution of parameter estimations obtained by MC simulation for $(\eta, \theta)=(2,2) /$ LS-WLS-ML. 
Table 4. Simulation results for the estimation of the parameters $(\eta, \theta)=(2,2)$.

\begin{tabular}{|c|c|c|c|c|c|c|c|}
\hline \multirow{2}{*}{ Pop. Size } & \multirow{2}{*}{ Method } & \multicolumn{3}{|c|}{$n=100$} & \multicolumn{3}{|c|}{$n=500$} \\
\hline & & Scale & Shape & def & Scale & Shape & def \\
\hline \multirow{7}{*}{50} & $\mathbf{L S}$ & 2,0201 & 1,8966 & 0,0648 & 2,0085 & 1,9699 & 0,0120 \\
\hline & WLS & 2,0071 & 1,9595 & 0,0547 & 2,0025 & 1,9945 & 0,0090 \\
\hline & ML & 1,9970 & 2,0258 & 0,0365 & 2,0016 & 2,0075 & 0,0070 \\
\hline & LS-WLS & 2,0104 & 1,9496 & 0,0340 & 2,0166 & 1,9814 & 0,1024 \\
\hline & LS-ML & 2,0039 & 1,9844 & 0,0286 & 2,0159 & 1,9875 & 0,0171 \\
\hline & WLS-ML & 1,9996 & 1,9984 & 0,0271 & 2,0030 & 1,9978 & 0,0069 \\
\hline & LS-WLS-ML & 1,9987 & 1,9809 & 0,0214 & 2,0024 & 1,9942 & 0,0045 \\
\hline \multirow{7}{*}{100} & LS & 2,0109 & 1,8982 & 0,0608 & 2,0095 & 1,9671 & 0,0128 \\
\hline & WLS & 1,9958 & 1,9624 & 0,0475 & 2,0032 & 1,9892 & 0,0099 \\
\hline & ML & 1,9892 & 2,0228 & 0,0361 & 2,0024 & 2,0050 & 0,0072 \\
\hline & LS-WLS & 2,0001 & 1,9517 & 0,0305 & 2,0047 & 1,9860 & 0,0063 \\
\hline & LS-ML & 1,9908 & 1,9843 & 0,0255 & 2,0035 & 1,9941 & 0,0059 \\
\hline & WLS-ML & 1,9915 & 1,9992 & 0,0274 & 2,0020 & 1,9999 & 0,0058 \\
\hline & LS-WLS-ML & 1,9907 & 1,9791 & 0,0207 & 2,0017 & 1,9932 & 0,0047 \\
\hline \multirow{7}{*}{250} & LS & 2,0227 & 1,9046 & 0,0638 & 2,0075 & 1,9629 & 0,0124 \\
\hline & WLS & 2,0074 & 1,9658 & 0,0492 & 2,0020 & 1,9901 & 0,0101 \\
\hline & ML & 2,0006 & 2,0298 & 0,0392 & 2,0002 & 2,0041 & 0,0073 \\
\hline & LS-WLS & 2,0103 & 1,9551 & 0,0331 & 2,0036 & 1,9849 & 0,0063 \\
\hline & LS-ML & 2,0017 & 1,9894 & 0,0271 & 2,0005 & 1,9931 & 0,0056 \\
\hline & WLS-ML & 2,0022 & 2,0019 & 0,0287 & 2,0008 & 1,9996 & 0,0059 \\
\hline & LS-WLS-ML & 2,0005 & 1,9827 & 0,0223 & 2,0006 & 1,9926 & 0,0046 \\
\hline \multirow{2}{*}{ Pop. Size } & \multirow{2}{*}{ Method } & \multicolumn{3}{|c|}{$n=1000$} & \multicolumn{3}{|c|}{$n=2500$} \\
\hline & & Scale & Shape & def & Scale & Shape & def \\
\hline \multirow{7}{*}{50} & LS & 2,0027 & 1,9759 & 0,0058 & 2,0030 & 1,9905 & 0,0023 \\
\hline & WLS & 1,9992 & 1,9905 & 0,0046 & 2,0096 & 1,9964 & 0,0701 \\
\hline & ML & 1,9984 & 1,9991 & 0,0034 & 2,0006 & 2,0021 & 0,0015 \\
\hline & LS-WLS & 2,0034 & 1,9823 & 0,0060 & 2,0106 & 1,9891 & 0,0379 \\
\hline & LS-ML & 2,0136 & 1,9827 & 0,0186 & 2,0136 & 1,9893 & 0,0123 \\
\hline & WLS-ML & 1,9993 & 1,9945 & 0,0034 & 2,0016 & 1,9986 & 0,0019 \\
\hline & LS-WLS-ML & 1,9992 & 1,9918 & 0,0026 & 2,0023 & 1,9974 & 0,0014 \\
\hline \multirow{7}{*}{100} & LS & 2,0041 & 1,9787 & 0,0062 & 2,0028 & 1,9908 & 0,0024 \\
\hline & WLS & 2,0006 & 1,9948 & 0,0050 & 2,0011 & 1,9993 & 0,0019 \\
\hline & ML & 2,0000 & 2,0011 & 0,0037 & 2,0006 & 2,0029 & 0,0015 \\
\hline & LS-WLS & 2,0021 & 1,9903 & 0,0033 & 2,0019 & 1,9963 & 0,0013 \\
\hline & LS-ML & 2,0012 & 1,9942 & 0,0032 & 2,0020 & 1,9983 & 0,0015 \\
\hline & WLS-ML & 2,0002 & 1,9990 & 0,0030 & 2,0007 & 2,0014 & 0,0012 \\
\hline & LS-WLS-ML & 2,0001 & 1,9950 & 0,0024 & 2,0006 & 1,9988 & 0,0009 \\
\hline \multirow{7}{*}{250} & LS & 2,0032 & 1,9794 & 0,0058 & 2,0024 & 1,9899 & 0,0023 \\
\hline & WLS & 1,9998 & 1,9960 & 0,0049 & 2,0007 & 1,9994 & 0,0020 \\
\hline & ML & 1,9989 & 2,0033 & 0,0034 & 2,0001 & 2,0023 & 0,0014 \\
\hline & LS-WLS & 2,0007 & 1,9921 & 0,0029 & 2,0011 & 1,9971 & 0,0011 \\
\hline & LS-ML & 1,9992 & 1,9962 & 0,0026 & 2,0003 & 1,9986 & 0,0011 \\
\hline & WLS-ML & 1,9993 & 2,0002 & 0,0028 & 2,0002 & 2,0012 & 0,0011 \\
\hline & LS-WLS-ML & 1,9992 & 1,9962 & 0,0022 & 2,0002 & 1,9988 & 0,0008 \\
\hline
\end{tabular}


Table 5. Simulation results for the estimation of the parameters $(\eta, \theta)=(2,3)$.

\begin{tabular}{|c|c|c|c|c|c|c|c|}
\hline \multirow{2}{*}{ Pop. Size } & \multirow{2}{*}{ Method } & \multicolumn{3}{|c|}{$n=100$} & \multicolumn{3}{|c|}{$n=500$} \\
\hline & & Scale & Shape & def & Scale & Shape & def \\
\hline \multirow{7}{*}{50} & LS & 2,0151 & 2,8683 & 0,1140 & 2,0055 & 2,9426 & 0,0236 \\
\hline & WLS & 2,0123 & 2,9428 & 0,1264 & 2,0044 & 2,9752 & 0,0306 \\
\hline & ML & 2,0060 & 3,0542 & 0,1006 & 2,0009 & 3,0005 & 0,0116 \\
\hline & LS-WLS & 2,0092 & 2,9294 & 0,0551 & 2,0040 & 2,9664 & 0,0136 \\
\hline & LS-ML & 2,0090 & 2,9779 & 0,0557 & 2,0042 & 2,9785 & 0,0147 \\
\hline & WLS-ML & 2,0032 & 3,0072 & 0,0483 & 2,0014 & 2,9897 & 0,0091 \\
\hline & LS-WLS-ML & 2,0036 & 2,9768 & 0,0354 & 2,0012 & 2,9842 & 0,0065 \\
\hline \multirow{7}{*}{100} & LS & 2,0173 & 2,8546 & 0,1149 & 2,0046 & 2,9484 & 0,0247 \\
\hline & WLS & 2,0081 & 2,9442 & 0,0820 & 2,0000 & 2,9910 & 0,0187 \\
\hline & ML & 2,0025 & 3,0489 & 0,0681 & 1,9996 & 3,0072 & 0,0131 \\
\hline & LS-WLS & 2,0104 & 2,9326 & 0,0496 & 2,0014 & 2,9800 & 0,0094 \\
\hline & LS-ML & 2,0047 & 2,9816 & 0,0404 & 2,0004 & 2,9903 & 0,0087 \\
\hline & WLS-ML & 2,0049 & 3,0019 & 0,0437 & 1,9998 & 2,9997 & 0,0101 \\
\hline & LS-WLS-ML & 2,0038 & 2,9716 & 0,0308 & 2,0001 & 2,9897 & 0,0066 \\
\hline \multirow{7}{*}{250} & LS & 2,0153 & 2,8439 & 0,1151 & 2,0033 & 2,9489 & 0,0228 \\
\hline & WLS & 2,0053 & 2,9367 & 0,0816 & 1,9988 & 2,9882 & 0,0180 \\
\hline & ML & 2,0005 & 3,0331 & 0,0582 & 1,9986 & 3,0046 & 0,0122 \\
\hline & LS-WLS & 2,0077 & 2,9262 & 0,0471 & 2,0000 & 2,9791 & 0,0092 \\
\hline & LS-ML & 2,0013 & 2,9776 & 0,0356 & 1,9990 & 2,9880 & 0,0082 \\
\hline & WLS-ML & 2,0015 & 2,9960 & 0,0398 & 1,9987 & 2,9980 & 0,0091 \\
\hline & LS-WLS-ML & 2,0013 & 2,9695 & 0,0277 & 1,9986 & 2,9881 & 0,0064 \\
\hline \multirow{2}{*}{ Pop. Size } & \multirow{2}{*}{ Method } & \multicolumn{3}{|c|}{$n=1000$} & \multicolumn{3}{|c|}{$n=2500$} \\
\hline & & Scale & Shape & def & Scale & Shape & def \\
\hline \multirow{7}{*}{50} & LS & 2,0028 & 2,9647 & 0,0125 & 2,0015 & 2,9830 & 0,0045 \\
\hline & WLS & 2,0002 & 2,9914 & 0,0094 & 2,0004 & 2,9979 & 0,0033 \\
\hline & ML & 1,9997 & 3,0014 & 0,0061 & 1,9999 & 3,0021 & 0,0022 \\
\hline & LS-WLS & 2,0033 & 2,9781 & 0,0124 & 2,0112 & 2,9829 & 0,0950 \\
\hline & LS-ML & 2,0069 & 2,9783 & 0,0202 & 2,0053 & 2,9877 & 0,0110 \\
\hline & WLS-ML & 2,0007 & 2,9908 & 0,0114 & 2,0004 & 2,9972 & 0,0040 \\
\hline & LS-WLS-ML & 2,0001 & 2,9909 & 0,0045 & 2,0008 & 2,9943 & 0,0022 \\
\hline \multirow{7}{*}{100} & LS & 2,0027 & 2,9689 & 0,0118 & 2,0021 & 2,9840 & 0,0044 \\
\hline & WLS & 1,9999 & 2,9982 & 0,0090 & 2,0006 & 2,9978 & 0,0036 \\
\hline & ML & 1,9996 & 3,0056 & 0,0063 & 2,0006 & 3,0007 & 0,0025 \\
\hline & LS-WLS & 2,0009 & 2,9889 & 0,0048 & 2,0013 & 2,9937 & 0,0019 \\
\hline & LS-ML & 2,0008 & 2,9939 & 0,0054 & 2,0013 & 2,9951 & 0,0022 \\
\hline & WLS-ML & 1,9997 & 3,0026 & 0,0047 & 2,0007 & 2,9989 & 0,0019 \\
\hline & LS-WLS-ML & 1,9998 & 2,9958 & 0,0033 & 2,0007 & 2,9965 & 0,0014 \\
\hline \multirow{7}{*}{250} & LS & 2,0039 & 2,9657 & 0,0111 & 2,0021 & 2,9846 & 0,0045 \\
\hline & WLS & 2,0015 & 2,9961 & 0,0085 & 2,0008 & 2,9982 & 0,0036 \\
\hline & ML & 2,0007 & 3,0050 & 0,0056 & 2,0005 & 3,0031 & 0,0025 \\
\hline & LS-WLS & 2,0023 & 2,9876 & 0,0043 & 2,0011 & 2,9944 & 0,0018 \\
\hline & LS-ML & 2,0010 & 2,9942 & 0,0038 & 2,0007 & 2,9972 & 0,0017 \\
\hline & WLS-ML & 2,0009 & 3,0016 & 0,0041 & 2,0006 & 3,0014 & 0,0019 \\
\hline & LS-WLS-ML & 2,0010 & 2,9942 & 0,0028 & 2,0006 & 2,9974 & 0,0013 \\
\hline
\end{tabular}


Table 6. Simulation results for the estimation of the parameters $(\eta, \theta)=(3,2)$.

\begin{tabular}{|c|c|c|c|c|c|c|c|}
\hline \multirow{2}{*}{ Pop. Size } & \multirow{2}{*}{ Method } & \multicolumn{3}{|c|}{$n=100$} & \multicolumn{3}{|c|}{$n=500$} \\
\hline & & Scale & Shape & def & Scale & Shape & def \\
\hline \multirow{7}{*}{50} & $\mathbf{L S}$ & 3,0235 & 1,8941 & 0,0764 & 3,0135 & 1,9644 & 0,0163 \\
\hline & WLS & 3,0015 & 1,9525 & 0,0656 & 3,0036 & 1,9909 & 0,0128 \\
\hline & ML & 2,9901 & 2,0229 & 0,0509 & 3,0028 & 2,0037 & 0,0106 \\
\hline & LS-WLS & 3,0103 & 1,9424 & 0,0492 & 3,0104 & 1,9823 & 0,0110 \\
\hline & LS-ML & 2,9954 & 1,9807 & 0,0399 & 3,0073 & 1,9915 & 0,0105 \\
\hline & WLS-ML & 2,9933 & 1,9985 & 0,0424 & 3,0031 & 1,9987 & 0,0087 \\
\hline & LS-WLS-ML & 2,9896 & 1,9757 & 0,0334 & 3,0034 & 1,9913 & 0,0076 \\
\hline \multirow{7}{*}{100} & LS & 3,0331 & 1,8945 & 0,0797 & 3,0063 & 1,9660 & 0,0149 \\
\hline & WLS & 3,0105 & 1,9565 & 0,0614 & 2,9976 & 1,9916 & 0,0128 \\
\hline & ML & 2,9988 & 2,0224 & 0,0510 & 2,9959 & 2,0043 & 0,0099 \\
\hline & LS-WLS & 3,0146 & 1,9489 & 0,0450 & 3,0001 & 1,9858 & 0,0087 \\
\hline & LS-ML & 3,0012 & 1,9828 & 0,0396 & 2,9969 & 1,9926 & 0,0081 \\
\hline & WLS-ML & 3,0015 & 1,9967 & 0,0397 & 2,9962 & 1,9993 & 0,0083 \\
\hline & LS-WLS-ML & 2,9992 & 1,9763 & 0,0330 & 2,9957 & 1,9918 & 0,0069 \\
\hline \multirow{7}{*}{250} & LS & 3,0367 & 1,9006 & 0,0756 & 3,0056 & 1,9621 & 0,0158 \\
\hline & WLS & 3,0165 & 1,9568 & 0,0625 & 2,9969 & 1,9910 & 0,0132 \\
\hline & ML & 3,0040 & 2,0260 & 0,0487 & 2,9944 & 2,0036 & 0,0101 \\
\hline & LS-WLS & 3,0200 & 1,9483 & 0,0442 & 2,9996 & 1,9851 & 0,0090 \\
\hline & LS-ML & 3,0041 & 1,9860 & 0,0366 & 2,9948 & 1,9917 & 0,0082 \\
\hline & WLS-ML & 3,0067 & 1,9998 & 0,0390 & 2,9954 & 1,9996 & 0,0084 \\
\hline & LS-WLS-ML & 3,0037 & 1,9776 & 0,0312 & 2,9949 & 1,9914 & 0,0069 \\
\hline \multirow{2}{*}{ Pop. Size } & \multirow{2}{*}{ Method } & \multicolumn{3}{|c|}{$n=1000$} & \multicolumn{3}{|c|}{$n=2500$} \\
\hline & & Scale & Shape & def & Scale & Shape & def \\
\hline \multirow{7}{*}{50} & LS & 3,0056 & 1,9790 & 0,0072 & 3,0025 & 1,9917 & 0,0030 \\
\hline & WLS & 3,0003 & 1,9963 & 0,0060 & 2,9995 & 1,9995 & 0,0025 \\
\hline & ML & 2,9990 & 2,0029 & 0,0048 & 2,9995 & 2,0020 & 0,0020 \\
\hline & LS-WLS & 3,0044 & 1,9900 & 0,0051 & 3,0043 & 1,9938 & 0,0039 \\
\hline & LS-ML & 3,0045 & 1,9944 & 0,0066 & 3,0066 & 1,9966 & 0,0069 \\
\hline & WLS-ML & 3,0008 & 1,9992 & 0,0041 & 3,0007 & 2,0005 & 0,0021 \\
\hline & LS-WLS-ML & 3,0000 & 1,9960 & 0,0033 & 3,0006 & 1,9983 & 0,0018 \\
\hline \multirow{7}{*}{100} & $\mathbf{L S}$ & 3,0066 & 1,9791 & 0,0075 & 3,0039 & 1,9891 & 0,0030 \\
\hline & WLS & 3,0004 & 1,9998 & 0,0063 & 3,0013 & 1,9987 & 0,0025 \\
\hline & ML & 2,9993 & 2,0047 & 0,0050 & 3,0004 & 2,0016 & 0,0019 \\
\hline & LS-WLS & 3,0023 & 1,9933 & 0,0042 & 3,0025 & 1,9959 & 0,0018 \\
\hline & LS-ML & 3,0007 & 1,9964 & 0,0043 & 3,0018 & 1,9980 & 0,0019 \\
\hline & WLS-ML & 2,9996 & 2,0022 & 0,0041 & 3,0008 & 2,0005 & 0,0016 \\
\hline & LS-WLS-ML & 2,9995 & 1,9964 & 0,0034 & 3,0009 & 1,9980 & 0,0014 \\
\hline \multirow{7}{*}{250} & LS & 3,0061 & 1,9760 & 0,0077 & 3,0032 & 1,9887 & 0,0030 \\
\hline & WLS & 3,0009 & 1,9937 & 0,0062 & 3,0005 & 1,9983 & 0,0025 \\
\hline & ML & 2,9991 & 2,0015 & 0,0050 & 2,9996 & 2,0014 & 0,0019 \\
\hline & LS-WLS & 3,0022 & 1,9898 & 0,0043 & 3,0013 & 1,9962 & 0,0017 \\
\hline & LS-ML & 2,9993 & 1,9946 & 0,0042 & 2,9999 & 1,9978 & 0,0016 \\
\hline & WLS-ML & 2,9994 & 1,9985 & 0,0041 & 2,9999 & 1,9999 & 0,0016 \\
\hline & LS-WLS-ML & 2,9991 & 1,9943 & 0,0034 & 2,9999 & 1,9978 & 0,0013 \\
\hline
\end{tabular}




\section{Application}

In this section, the application of parameter estimation methods to the real data set is investigated with the help of NSGA-II algorithm. Monthly measured wind speed data of Basel/Switzerland, consisting of 743 observations, were used as a data set.

The Kolmogorov-Smirnov goodness of fit test was used to test the suitability of the data set to the two-parameter Weibull distribution. According to KolmogorovSmirnov test statistic value 0.04702 ( $p$ value: 0.07252 ), the Weibull distribution could be an appropriate model for fitting these data. In this study, $\alpha=0.05$ is taken.

The best solution among Pareto points in the parameter space for LS-WLS, LS-ML, WLS-ML and LS-WLS-ML given in Figures 9 and 10 is obtained by choosing the point corresponding to the best $\log \mathrm{L}$ value of the prediction points.

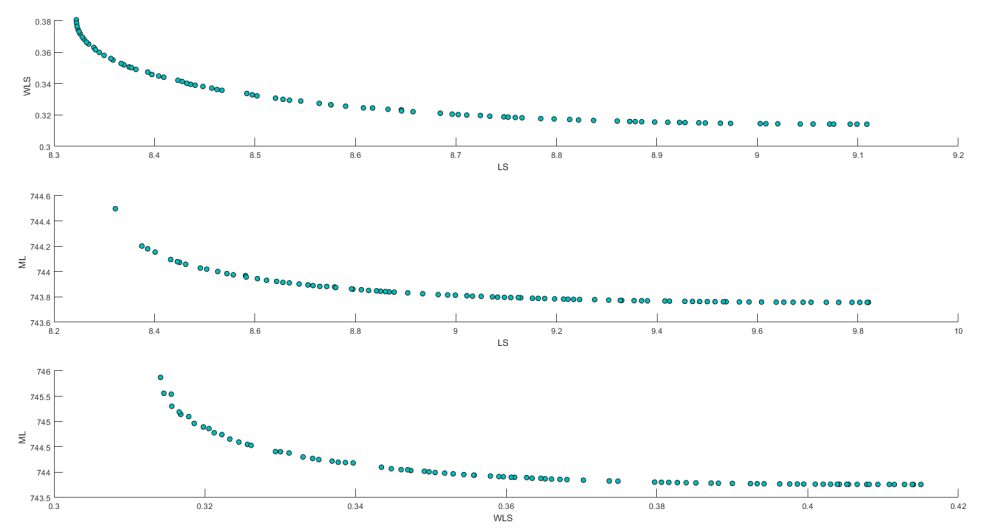

Figure 9. Pareto points in parameter space for LS-WLS, LS-ML and WLS-ML.

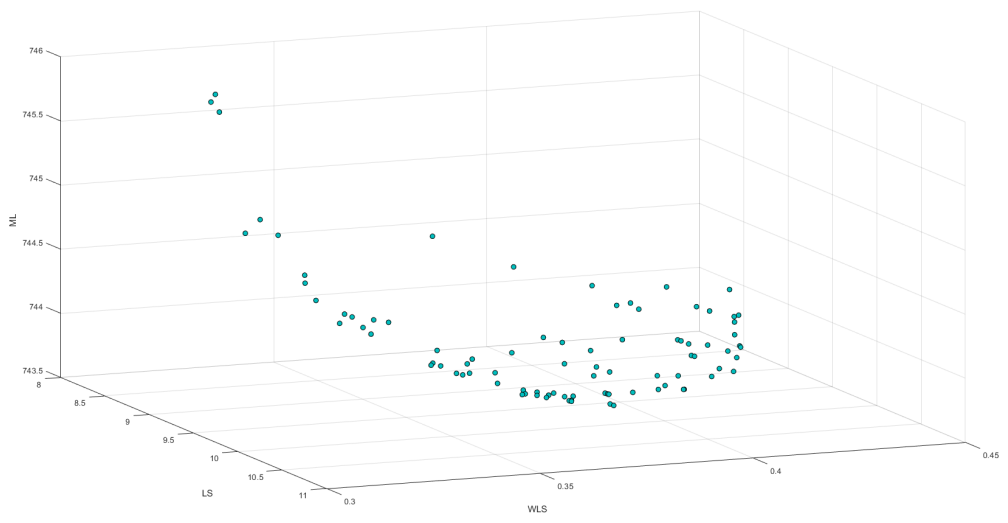

Figure 10. Pareto points in parameter space for LS-WLS-ML.

Parameter estimation values, $\log \mathrm{L}$ and Akaike information criterion (AIC) for the considered methods are given in Table 7. It is clear that ML, LS-ML, WLS-ML and LS-WLSML have the largest $\log \mathrm{L}$ and also the smallest AIC values. Therefore, these cases make the best parameter estimation besides giving trustworthy results. See also Figure 11, in which a histogram and fitted densities are given. 
Table 7. Parameter estimation values, $\log \mathrm{L}$ and AIC for wind speed dataset.

\begin{tabular}{|c|c|c|c|c|}
\hline Method & Scale & Shape & $\log L$ & AIC \\
\hline LS & 13,5795 & 1,7414 & $-2437,8630$ & 4879,7259 \\
WLS & 13,8614 & 1,7277 & $-2439,2178$ & 4882,4355 \\
ML & 13,5472 & 1,7999 & $-2437,1186$ & 4878,2372 \\
LS-WLS & 13,5795 & 1,7414 & $-2437,8637$ & 4879,7274 \\
LS-ML & 13,5475 & 1,7999 & $-2437,1186$ & 4878,2372 \\
WLS-ML & 13,5475 & 1,7999 & $-2437,1186$ & 4878,2372 \\
LS-WLS-ML & 13,5464 & 1,7997 & $-2437,1186$ & 4878,2373 \\
\hline
\end{tabular}

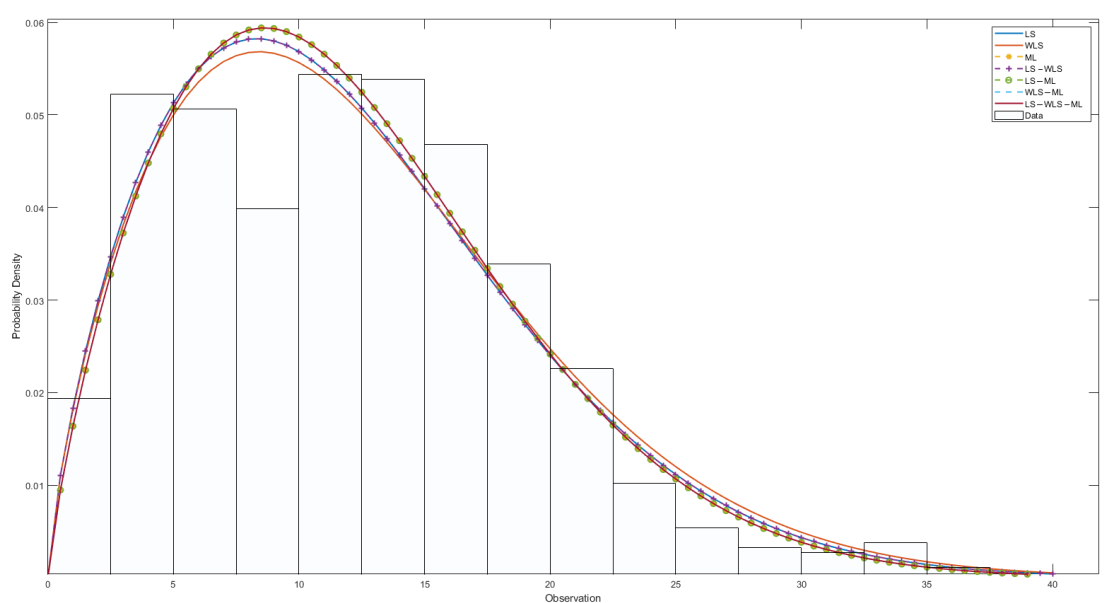

Figure 11. Histogram and fitted densities for wind speed dataset.

\section{Conclusion}

In this study, based on ML and LS estimation methods, for the parameter estimation of the two-parameter Weibull distribution, a multi-objective programming approach, is proposed in which ML and LS methods are evaluated together in the estimation process. NSGA-II method, which is a multi-objective heuristic optimization method, is used to determine the variable values that will give the best value to the objective functions in multi-objective optimization problems. Genetic Algorithm-based NSGA-II is one of the most effective methods used in obtaining the Pareto solution set, and it reaches real Pareto values faster by obtaining different solutions in the Pareto solution set due to its features such as fast non-dominant sorting and crowding distance.

A comprehensive MC simulation study is conducted to test the performance of this proposed approach. The simulation results show that the WLS-ML case makes the best parameter estimation in most cases. In addition, a real data set was analyzed to show the applicability of the proposed approach and gave the best estimation results in ML, LS-ML and LS-WLS-ML cases as well as the WLS-ML case. Especially, the results show that this proposed multi-objective programming approach is effective for estimating the parameters of the two parameter Weibull distribution with respect to deficiency criteria.

As a future research, the proposed approach can be applied to estimate the parameters of different statistical distributions.

\section{References}

[1] B. Abbasi, A. Jahromi, J. Arkat and M. Hosseinkouchack, Estimating the parameters of Weibull distribution using simulated annealing algorithm, Appl. Math. Comput. 183 (1), 85-93, 2006. 
[2] B. Abbasi, S. Niaki, M. Khalife and Y. Faize, Hybrid variable neighborhood search and simulated annealing algorithm to estimate the three parameters of the Weibull distribution, Expert Syst. Appl. 38 (1), 700-708, 2011.

[3] Ş. Acıtaş, Ç.H. Aladağ and B. Şenoğlu, A new approach for estimating the parameters of Weibull distribution via particle swarm optimization: An application to the strengths of glass fibre data, Reliab. Eng. Syst. 183, 116-127, 2019.

[4] B. Bergman, Estimation of Weibull parameters using a weight function, J. Mater. Sci. Lett. 5 (6), 611-614, 1986.

[5] Y.K. Chu and J.C. Ke, Computation approaches for parameter estimation of Weibull distribution, Math. Comput. Appl. 17 (1), 39-47, 2012.

[6] K.C. Datsiou and M. Overend, Weibull parameter estimation and goodness of fit for glass strength data, Struct. Saf. 73, 29-41, 2018.

[7] I.J. Davies, Unbiased estimation of the Weibull scale parameter using linear least squares analysis, J. Eur. Ceram. Soc. 37 (8), 2973-2981, 2017.

[8] K. Deb, Multi-Objective Optimization Using Evolutionary Algorithms, John-Wiley and Sons, 2004.

[9] K. Deb, A. Pratap, S. Agarwal and T. Meyarivan, A fast and elitist multi-objective genetic algorithm: NSGA-II, IEEE Trans. Evol. Comput. 6 (2), 182-197, 2002.

[10] A. Hossain and H. Howlader, Unweighted least squares estimation of Weibull parameters, J. Stat. Comput. Simul. 54 (1-3), 265-271, 1996.

[11] Y. Lei, Evaluation of three methods for estimating the Weibull distribution parameters of Chinese pine (Pinus tabulaeformis), J. For. Sci. 54 (12), 566-571, 2008.

[12] R. Luus and M. Jammer, Estimation of parameters in 3-parameter Weibull probability distribution functions, Hung. J. Ind. Chem. 33 (1-2), 69-73, 2005.

[13] D. Markovic, D. Jukic and M. Bensic, Nonlinear weighted least squares estimation of a three-parameter Weibull density with a nonparametric start, J. Comput. Appl. Math. 228 (1), 304-312, 2009.

[14] M. Nassar, A.Z. Afify, S. Dey and D. Kumar, A new extension of Weibull distribution: Properties and different methods of estimation, J. Comput. Appl. Math. 336, 439-457, 2018.

[15] H.H. Örkcü, V.S. Özsoy, E. Aksoy and M.. Doğan, Estimating the parameters of 3-p Weibull distribution using particle swarm optimization: A comprehensive experimental comparison, Appl. Math. Comput. 268, 201-226, 2015.

[16] I. Pobacikova and Z. Sedliackova, Comparison of four methods for estimating the Weibull distribution parameters, Appl. Math. Sci. 8 (83), 4137-4149, 2014.

[17] M.L. Tiku and A.D. Akkaya, Robust Estimation and Hypothesis Testing, New Age International (P) Ltd. Publishers, 2004.

[18] X.S. Yang, Engineering Optimization: An Introduction with Metaheuristic Applications, John Wiley and Sons, 2010. 